ITFA-2004-29

\title{
To be or not to be? Magnetic monopoles in non-abelian gauge theories ${ }^{1}$
}

\author{
F. Alexander Bais ${ }^{2}$ \\ Institute for Theoretical Physics \\ University of Amsterdam
}

\begin{abstract}
Magnetic monopoles form an inspiring chapter of theoretical physics, covering a variety of surprising subjects. We review their role in nonabelian gauge theories. An exposé of exquisite physics derived from a hypothetical particle species, because the fact remains that in spite of ever more tempting arguments from theory, monopoles have never reared their head in experiment. For many relevant particulars, references to the original literature are provided.
\end{abstract}

\section{Introduction}

"Under these circumstances one would be surprised if Nature had made no use of it"

P.A.M. Dirac (1931)

The homogeneous Maxwell equations

$$
\nabla \cdot \mathbf{B}=0, \quad \nabla \times \mathbf{E}+\frac{\partial \mathbf{B}}{\partial t}=0
$$

have no source terms, reflecting the plain fact that isolated magnetic poles or magnetic currents have never been observed in nature. The experimental limit for observing heavy (non-relativistic) monopoles in cosmic rays is presently well below $10^{-15} \mathrm{~cm}^{-2} \mathrm{sr}^{-1} \mathrm{sec}^{-1}$, whereas on the other hand accelerator searches have not produced any candidate up to masses of well over $500 \mathrm{GeV} / \mathrm{c}^{2}$. From a theoretical point of view this is surprising because the absence of monopoles introduces an asymmetry in the equations for which there does not appear to be any intrinsic reason. On the contrary, when Dirac in his seminal 1931 paper [1], introduced the magnetic monopole and studied the consequences of its existence in the context of ordinary quantum mechanics he did the striking discovery that the product of electric and magnetic charges had to be quantized (with $\hbar=c=1$ ),

$$
e g=2 \pi n,
$$

\footnotetext{
${ }^{1}$ Contribution to "Fifty Years of Yang-Mills Theory", edited by G.'t Hooft

2E-mail: bais@science.uva.nl
} 
implying that the existence of a single monopole would explain the observed quantization of all electric charges. In his paper he introduces the magnetic "Dirac" potential,

$$
\begin{aligned}
e \mathbf{A}^{D}(\mathbf{r}) & =\frac{e g}{4 \pi} \mathbf{a}_{\hat{n}}(\mathbf{r}) \\
\mathbf{a}_{\hat{n}}(\mathbf{r}) & =\frac{\hat{\mathbf{r}} \times \hat{\mathbf{n}}}{r(1-\hat{\mathbf{r}} \cdot \hat{\mathbf{n}})}
\end{aligned}
$$

which has besides the obvious singularity at the origin also a string singularity extending from the origin out along the $\hat{\mathbf{n}}$ direction. It is the requirement that physical charges should not be able to detect the string that enforces the quantization condition. The quantization and conservation of magnetic charge in electrodynamics has a topological origin and is related to the existence of nontrivial circle bundles over the two-sphere

$$
\begin{array}{cc}
U(1) \hookrightarrow & B \\
& \downarrow \\
& S^{2}
\end{array}
$$

These bundles are classified by the homotopy classes of of mappings of a circle in the gauge group $U(1)$. It follows also directly from the argument given by Dirac that the different allowed magnetic charges are indeed in one to one correspondence with these classes. The simplest nontrivial example is the case $B=S^{3}$, corresponding to the so-called Hopf fibration introduced by the Hopf [2] in 1931, the same year in which Dirac wrote his monopole paper. This correspondence explains the topological nature of magnetic charge and its conservation; the addition or composition of charges is given by the homotopy group $\pi_{1}(U(1)) \simeq \pi_{1}\left(S^{1}\right)=\mathbb{Z}$. This topological argument may be extended to classify the Dirac-type monopoles in theories with non-abelian groups. For example in a pure gauge theory (with only adjoint fields) the group is the simply connected covering group divided by its center, e.g. $G \simeq S U(N) / \mathbb{Z}_{N}$ and consequently $\pi_{1}(G)=\mathbb{Z}_{\mathbb{N}}$, implying that Dirac-type monopole charges are only conserved modulo $N$. In spite of its attractive simplicity and elegance, the Dirac analysis is vulnerable because monoples have to be introduced by hand. A hypothetical particle which remains elusive to this very day.

In the theoretical arena however, monopoles thrived for the more then 70 years that followed. The subject certainly went through various ups and downs but it is fair to say that in spite of the dramatic lack of experimental evidence, the theoretical case has gained strength to the point that monopoles appear to be unavoidable in any theory that wants to truly unify electromagnetism with the other fundamental forces. Whether it is through the road of Grand Unification or through the compactification of spatial dimensions á la Kaluza and Klein and/or through String Theory, monopoles appear to be the price one has to pay.

A magnificent impetus to the subject was the remarkable discovery of 't Hooft and Polyakov 3 , 4 that in non-abelian gauge theories spontaneously broken down to some $U(1)$ subgroup, magnetic monopoles appear as solitons, i.e. 
as regular, finite energy solutions to the field equations. Monopoles reappeared as natural and unavoidable inhabitants of the non-abelian landscape; a package deal. And again the conservation of magnetic charge arose as a consequence of the topology of the solution space and not because of some symmetry argument.

After introducing some monopole essentials we discuss a variety of topics that make monopole physics so fascinating, varying from charge quantization issues and monopole stability, to making fermions out of bosons. From theta angle physics to the catalysis of baryon decay by Grand Unified Monopoles. From quantum moduli to the links with a myriad of integrable systems...

Quantum Chromo Dynamics is a different domain where monopoles have been used extensively in particular to explain the confinement phenomenon. Here the challenge is to show that the vacuum consist of monopoles that condensed to form a magnetically superconducting ground state which confines quarks, as was suggested by Mandelstam and 't Hooft. This is a statement that monopoles are literally everywhere! How paradoxical nature can get? We will return to this point when discussing the work of Seiberg and Witten on $\mathrm{N}=2$ supersymmetric Yang-Mills Theory.

\section{Monopoles in Yang-Mills-Higgs Theories}

We consider spontaneously broken gauge theory - or the Yang-Mills-Higgs system, with a gauge group $G$ and a Higgs field $\Phi$ typically in the adjoint representation of the group. We write the Lagrangian ${ }^{3}$

$$
\mathcal{L}=-\frac{1}{4} F_{\mu \nu}^{2}+\left(D_{\mu} \Phi\right)^{2}-\lambda V(\Phi)
$$

where

$$
D_{\mu} \Phi=\partial_{\mu}+e\left[A_{\mu}, \Phi\right] .
$$

The symmetry is broken to a subgroup $H \subset G$ by a vacuum expectation value $<\Phi>=\Phi_{0}$. For the solutions one imposes the asymptotic condition at spatial infinity that each of the terms vanishes sufficiently fast to have an integrable energy density. This implies that $\Phi(\infty) \approx \Phi_{0}$ but also that $D_{\mu} \Phi(\infty) \approx 0$. We may also conclude that

$$
\left[D_{\mu}, D_{\nu}\right] \Phi \approx 0
$$

which implies that the only allowed long range (Coulombic type) components for the gauge field $F_{\mu \nu} \propto\left[D_{\mu}, D_{\nu}\right]$ have to lie in the unbroken part $H$.

\subsection{The BPS limit}

In the Lagrangian (3) one may set the parameter $\lambda=0$, which corresponds to the so-called Bogomol'nyi-Prasad-Sommerfeld (BPS) limit. In this limit a lower

\footnotetext{
${ }^{3}$ Traces over squares of generators are implied but not explicitly indicated if obvious. Spatial vector quantities are printed in boldface
} 
bound on the energy for a static, purely magnetic solution can be derived [], the expression for the energy can be casted in the form

$$
E=\frac{1}{2} \int[\mathbf{B} \mp \mathbf{D} \Phi]^{2} d^{3} x \pm \int \mathbf{B} \cdot \mathbf{D} \Phi d^{3} x .
$$

The last term can after a partial integration and using the Bianchi identity be rewritten $\mathbf{D B}=0$ as,

$$
\pm \int \mathbf{B} \cdot \mathbf{D} \Phi d^{3} x=\left|g \Phi_{0}\right|=\left(\frac{e g}{4 \pi}\right)\left(\frac{4 \pi}{e^{2}}\right) M_{w},
$$

where $M_{w}=\left|e \Phi_{0}\right|$ is the mass of the charged vector particles. This calculation shows that the mass of the monopole is large: typically the mass scale in the theory divided by the fine structure constant. The lower bound in (6) is saturated if the fields satisfy the first order Bogomol'nyi equations

$$
\mathbf{B}= \pm \mathbf{D} \Phi
$$

This system of non-linear partial differential equations has been studied extensively and has become a most exquisite and rich laboratory for mathematical physics, in particular with respect to the study of higher dimensional integrable systems. We return to this topic towards the end of the Chapter.

\subsection{The 't Hooft-Polyakov Monopole}

In 1974 't Hooft and Polyakov wrote their famous papers on the existence of a regular magnetic monopole solution in the Georgi-Glashow model with $G=$ $S O(3)$ broken down to $U(1)$ by a Higgs field in the triplet representation. Their argument implied the existence of such regular monopoles in any unified gauge theory where the $U(1)$ of electromagnetism would be a subgroup of some simple non-abelian group. The crucial difference with the original Dirac proposal was that, because these monopoles appear as regular, soliton-like solutions to the classical field equations, they are unavoidable and cannot be left out. It is amusing to note that it was originally thought that unifying electromagnetism in a larger compact non-abelian group would explain the quantization of electric charge without the need for magnetic monopoles. This basically because the charge generator is identified with a compact $U(1)$ generator of the unified group. With the discovery of the regular monopoles the two ways to obtain charge quantization boil down to one and the same argument. 't Hooft and Polyakov wrote down a spherically symmetric ansatz with respect to the mixed angular momentum generator $\mathbf{J}=\mathbf{L}+\mathbf{T}$, where $\mathbf{L}=-i \mathbf{r} \times \nabla$ is the ordinary spatial part of angular momentum and $\mathbf{T}$ stands for the generators of the gauge group. The symmetric ansatz obeys the following natural conditions:

$$
\begin{aligned}
{\left[J_{i}, \Phi\right] } & =0 \\
{\left[J_{i}, A_{j}\right] } & =i \varepsilon_{i j k} A_{k}
\end{aligned}
$$




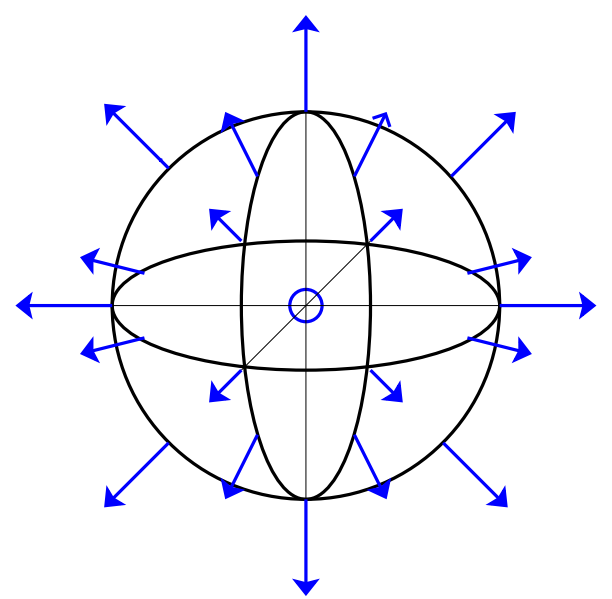

Figure 1: The topology of the isovector Higgs field for the 't Hooft-Polyakov monopole configuration, where the orientation in isospace is aligned with the position vector in real space.

and takes the form

$$
\begin{aligned}
\Phi & =\frac{h(x)}{x}(\hat{\mathbf{r}} \cdot \mathbf{T}) \\
\mathbf{A} & =\frac{1-k(x)}{x}(\hat{\mathbf{r}} \times \mathbf{T})
\end{aligned}
$$

where we used the dimensionless variable $x \equiv$ efr with $f \equiv\left|\Phi_{0}\right|$. Prasad and Sommerfield found an exact solution [6] in the BPS limit named after them.

$$
h(x)=1-x \operatorname{coth} x, \quad k(x)=\frac{x}{\sinh x} .
$$

It was later pointed out by Bogomol'nyi that these expressions are a solution to a beautiful and much simpler system of the first order equations (8). Calculating the magnetic charge by integrating the unbroken radial magnetic field component at infinity, the intriguing result $g=4 \pi / e$ came out - twice the Dirac value. On the other hand, realizing that the monopole solution could also be written down in a theory with fields in doublet representations makes it less surprising, because these fields carry $q= \pm e / 2$. Dirac's veto is respected by all charges in the theory.

\section{Charge quantization in non-abelian theories}

We remarked before that long range fields are allowed only in the unbroken part of the gauge group. Simple time independent solutions that for large $r$ have a 
purely magnetic monopole field are

$$
e A=\frac{e g}{4 \pi}(1+\cos \theta) d \varphi+O\left(\frac{1}{r^{2}}\right)
$$

where the quantity eg/ $4 \pi$ is a constant element in the Lie algebra of $H$, i.e. just a Dirac type potential for any component in the unbroken group.

The extension of the Dirac argument to the general non-abelian case is rather straightforward. As the electrically charged fields that may appear in the theory form representations of $\mathrm{G}$ one has to impose that these are single valued if acted upon by the group element expeg. This quantization condition can be solved in terms of the simple roots $\vec{\gamma}_{i}$ (with $i=1, \ldots, r$ and $r$ is rank of $H$ ) of the root system of $H$. From the simple roots one constructs a convenient basis $\left\{C_{i}\right\}$ for

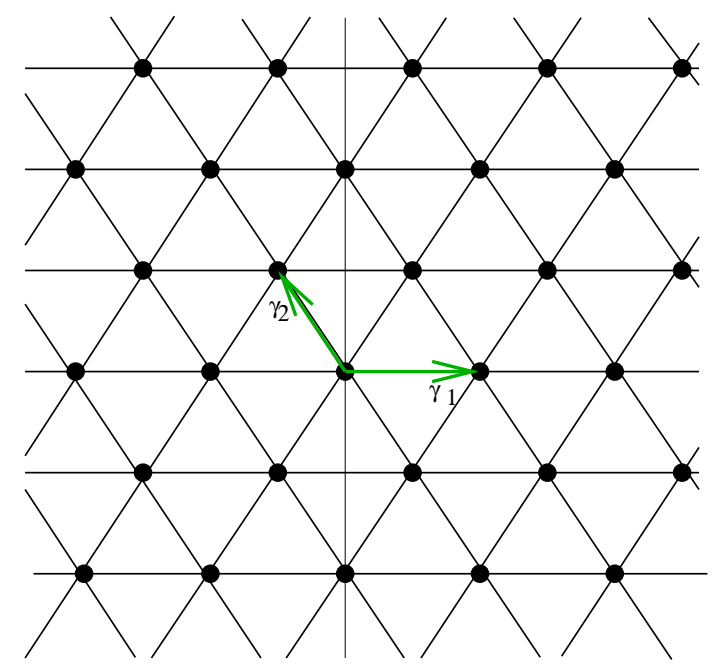

Figure 2: The Lattice of allowed magnetic charges for the group $S U(3)$ spanned by the (inverse) simple roots.

the commuting generators of the Cartan subalgebra of $H$ with the property that each basis element has per definition half integral eigenvalues when acting on a basis vector of any representation, this is achieved by defining,

$$
C_{i} \equiv \frac{\vec{\gamma}_{i}}{\left|\vec{\gamma}_{i}\right|^{2}} \cdot \vec{C}
$$

where $\{\vec{C}\}$ is the familiar Weyl basis for the Cartan subalgebra. The solution to the quantization condition takes the simple form:

$$
\frac{e g}{4 \pi}=\sum^{r} n_{i} C_{i}
$$

This solution can be represented as a $r$-dimenional lattice dual to the weight lattice of the group [7. For the simple example of $S O(3)$ the rank is $r=1$ and 
one gets indeed the charges which are $e g=4 \pi n$. For the group $S U(3)$ the corresponding magnetic charge lattice is given in Fig 2 In general the dual weight lattice may be thought of as the root lattice of a dual group and this observation led Goddard, Olive and Nuyts to suggest the existence of such a hidden dual symmetry group $[8$. We note that the existence of nonsingular solutions or even the asymptotic stability of the allowed charges does of course not follow from the quantization condition.

\subsection{Topological Charges}

Magnetic charges are conserved for topological reasons which implies that some suitably defined lowest allowed charges should be stable. It was recognized early on that the magnetic charge in non-abelian gauge theories could be related to some topological invariant defined by the asymptotic behavior of the Higgs field. The asymptotic conditions allow us to consider the Higgs field as a mapping from a closed surface at spatial infinity $\partial M$ into the gauge orbit of the particular vacuum solution $\Phi_{0}$, this orbit or vacuum manifold is homeomorphic to the coset space $G / H$.Well known are Coleman's Erice lectures 9 on the subject, which concerning this particular topic were based on earlier papers a number of Russian authors 10, 11, 12. So from

$$
\Phi(\infty): \partial M \rightarrow G / H
$$

it follows that these maps fall into inequivalent classes which form the second homotopy group of the coset space denoted as $\pi_{2}(G / H)$. These homotopy groups specify the composition rules for the topological charges in a quite general way, and it is worth saying a few things about them. The topological structure resides in the fiber bundle

$$
\begin{array}{ccc}
H \hookrightarrow & G \\
& \downarrow \\
& G / H
\end{array}
$$

One may determine the homotopy structure by exploiting the following exact sequence of mappings of homotopy groups:

$$
\cdots \rightarrow \pi_{2}(G) \rightarrow \pi_{2}(G / H) \rightarrow \pi_{1}(H) \rightarrow \pi_{1}(G) \rightarrow \pi_{1}(G / H) \rightarrow \cdots
$$

The exactness of the sequence refers to the fact that the image of a given homomorphism equals the kernel of the next one in the sequence. A theorem of Poincaré states that for all semisimple groups $\pi_{2}(G)=0$, in which case the exactness of the sequence implies that

$$
\pi_{2}(G / H) \simeq \operatorname{Ker}\left[\pi_{1}(H) \rightarrow \pi_{1}(G)\right]
$$

If $G$ is simply connected $\left(\pi_{1}(G)=0\right)$ the topological classification boils down to the fundamental group of the unbroken gauge group $H$, very much in line 
with the generalized Dirac-argument of the previous section. This may be understood as follows, we take a simply connected gauge group and choose $\Phi_{0}$ to break the symmetry to the maximal torus $H=U(1)^{\otimes r} \subset G$, then one obtains that $\pi_{1}(H)=\mathbb{Z}^{\otimes r}$. In this case all allowed charges on the dual weight lattice are topologically conserved. There is a perfect matching between topological sectors and points on the lattice determined by the quantization condition. The situation for the 't Hooft-Polyakov case is an example of this but there is one subtlety, because the gauge group $S O(3)$ is not simply connected - $\pi_{1}(S O(3))=\mathbb{Z}_{2}$, only the even elements of $\pi_{1}(H)$ are in the Kernel of the homomorphism of Eqn. (16). This explains that in spite of the fact that the the residual group is just $U(1)$ the quantization condition is twice the one given by Dirac.

Topologically stable monopoles do not occur in the electro-weak sector of the standard model because in the breaking of $G=S U(2) \times U(1)$ by a complex doublet one finds that $G / H \simeq S^{3}$ and consequently $\pi_{2}\left(S^{3}\right)=0$.

The situation changes drastically if the gauge group is only partially broken, then one may expect that there are fewer topologically conserved components to the magnetic charge. With a single adjoint Higgs field one may break for example to a group $H=U(1) \otimes K$ where $\mathrm{K}$ is semi-simple and simply connected in which case $\pi_{1}(H)=\mathbb{Z}$ and there is only a single component of the magnetic charge that is topologically conserved. This is the situation that arises in most Grand Unified Theories, for example the $S U(5)$ theory broken down to $S U(3) \times$ $S U(2) \times U(1)$. Monopoles with unbroken non-abelian symmetries are still rather poorly understood. The monopoles are to be related with conjugacy classes and the dyonic states do not fill complete representations of the unbroken group, but only representations of the centralizer of the magnetic charge vector. There is an obstruction to implement the full unbroken group in the presence of a magnetic charge - sometimes referred to as the problem of implementing global color 13 [14. Whereas for a single monopole the issue is quite well understood, if one is to study the multi-monopole sectors and the fusion properties of these monopoles the situation appears far from trivial[15].

A final nontrivial class of topological magnetic charges arises if the gauge group gets broken to non-simply-connected non-abelian subgroups in which case the magnetic charge is only additively concerved modulo some integer 16. With a Higgs field in the 6-dimensional representation of $S U(3)$ one may break $S U(3)$ to $S O(3)$ (defined by the embedding where the triplet of $S U(3)$ goes into the vector of $S O(3))$, then one obviously gets $\pi_{1}(S O(3))=\mathbb{Z}_{2}$. The resolution to the mismatch between the sets of allowed and topologically conserved charges has to be that certain components of the magnetic charge vectors on the dual weight lattice are unstable. This will be discussed in the following subsection.

Other homomorphisms in the exact homotopy sequence (15) have also physical content, for example to determine what happens when monopoles would cross a phase boundary [17. One can imagine monopoles which are formed at an early stage of the universe and one would then like to know their fate if the universe subsequently goes through various phase transitions. For example if one has two broken phases with $G \subset H_{1} \subset H_{2}$, the question is what happens to the monopoles that are allowed in the $H_{1}$ phase when they cross a boundary 
to the $H_{2}$ phase; will they be confined, converted or just decay in the vacuum? The exact sequence tells us for example that

$$
\operatorname{Im}\left[\pi_{1}\left(H_{2}\right) \rightarrow \pi_{1}\left(H_{1}\right)\right]=\operatorname{Ker}\left[\pi_{1}\left(H_{1}\right) \rightarrow \pi_{1}\left(H_{1} / H_{2}\right)\right]
$$

Given the fact that $\pi_{1}\left(H_{1} / H_{2}\right)$ labels the topological magnetic flux tubes in the $H_{2}$ phase the above equation determines exactly which $H_{1}$ monopoles will be confined.

\subsection{Charge instabilities, a mode analysis}

In general there is a discrepancy between the lattice of allowed magnetic charges defined by (12) and the topologically conserved (if not stable) subset. These should somehow be related to each other. One way to get insight in this question is to study the asymptotic stability of the long range magnetic Coulomb field of a charge on the lattice. Brandt and Neri studied the fluctuations and did indeed find certain unstable modes on this singular background [18. Let us expand

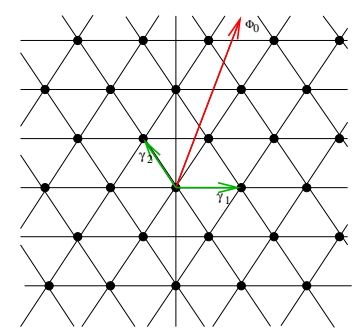

(a)

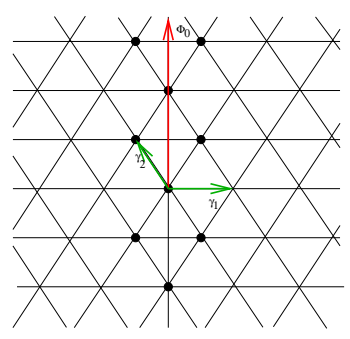

(b)

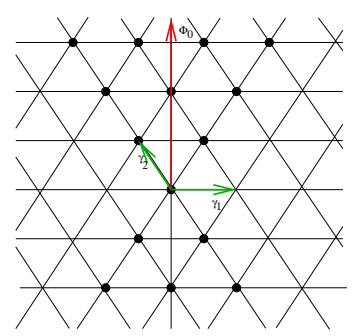

(c)

Figure 3: The lattice of charges allowed by the quantisation condition, spanned by the (inverse) simple roots,. In this figure also the direction of the Higgsfield $\Phi_{0}$ in the Cartan subalgebra is indicated. In (a) the stable charges for an arbitrary non-degenerate orientation of the Higgs field are indicated by black dots. In that case the residual gauge group is $U(1) \times U(1)$ and all allowed charges are topologically conserved. In (b) the Higgs field is degenerate and leaves the non-abelian group $U(2)$ unbroken. Now only one component of the magnetic charge is conserved, and in each topological sector only the smallest total charge is conserved. The points symmetric with respect to the Higgs field are gauge conjugates. In (c) we have indicated the same orientation of the Higgs field, but we have taken the Bogomoil'nyi limit and see that more charges are stable then one would expect on purely topological grounds. The horizontal quantum number is sometimes referred to as the holomorphic charge.

the Lie algebra valued fields around their classical backgrounds as:

$$
\begin{aligned}
e \mathbf{A} & =e \mathbf{A}_{D}+e \mathbf{a} \\
\Phi & =\Phi_{0}+\phi
\end{aligned}
$$

where $\mathbf{A}_{D}$ is the Dirac monopole potential and work in the background gauge $\mathbf{D} \cdot \mathbf{a}+[\Phi, \phi]=0$. It is convenient to expand the commutator of the fluctuation field with the monopole background and write,

$$
\left[\frac{e g}{4 \pi}, \mathbf{a}\right]=\sum_{\alpha} q(\alpha) \mathbf{a}^{\alpha} T_{\alpha}
$$


from which it follows that $q(\alpha)=0$ if $T_{\alpha}$ is a generator of the little group $S$ of the magnetic charge generator $e g / 4 \pi$. Note that $S \cap H \neq 0$. In the BPS limit we should replace $\Phi_{0}$ in the expansion (18) by $\Phi_{0}-e g / 4 \pi r$ which yields extra $q$ dependent terms from commutators with $\Phi$. The linearized fluctuation equations take the following form:

$$
\begin{aligned}
(\mathbf{D} \cdot \mathbf{D}) \mathbf{a}+\frac{2 q}{r^{2}}(\hat{\mathbf{r}} \times \mathbf{a})-\frac{2 q}{r^{2}} \hat{\mathbf{r}} \phi-\frac{q^{2}}{r^{2}} \mathbf{a}-m^{2} \mathbf{a} & =-E^{2} \mathbf{a} \\
(\mathbf{D} \cdot \mathbf{D}) \phi-\frac{2 q}{r^{2}} \hat{\mathbf{r}} \mathbf{a}-\frac{q^{2}}{r^{2}} \phi-m^{2} \phi & =-E^{2} \phi
\end{aligned}
$$

In these equations both fields carry a Lie algebra index $\alpha$ which is suppressed. The structure of this coupled system is now as follows, the terms which are dependent on $q=q(\alpha)$ are zero for the components which generate $S$, whereas the mass terms $m^{2}=m^{2}(\alpha)$ vanish for the components which generate $H$. For components of the fluctuation fields outside $H \cup S$ there will be no unstable modes because $E^{2}>0$. In the generic case, not the BPS limit, all q dependent terms vanish except the second term in the vector equation. This means that the equations decouple in that case and one may verify that the scalar perturbation has no unstable modes. The vector perturbations however turn out to have one unstable mode for components inside $S$ provided $|q| \geq 1$. In the generic case one arrives therefore at an important conclusion which reconciles the notion of topological charge and dynamical stability, namely, that in each topological class only the smallest total magnetic charge is stable. The stability analysis in the BPS limit is much more involved, indeed in that case there may be more than a single stable monopole in a given topological sector. We have illustrated various situations for $S U(3)$ in Fig 3 In the first figure (a) we give the complete lattice of allowed charges in $S U(3)$ all these are topologically conserved if one breaks $S U(3)$ to $U(1) \times U(1)$. The second figure (b) shows what happens if one breaks to $U(2)$ (with a Higgs field along the $\lambda_{8}$ direction), then the asymptotic analysis shows that only the black dots survive while the others have become unstable. Indeed the minimal total magnetic charge within each topological class survives, indicating an instability in the horizontal direction. The last figure (c) shows

what happens in the Bogomol'nyi limit where the stability analysis is affected by the massless scalar degrees of freedom, now the stable monopoles fill out a Weyl chamber around the Higgs direction.

\section{Cheshire charge and core instabilities}

It may happen that the topology of the vacuum manifold is more complicated than the ones we just discussed. In particular it may be such, that different types of defects can coexist. If the residual symmetry group is non-abelian these defects may have topological interactions, interactions not mediated by the exchange of particles, but interactions that are essentially of a kinematical nature. Yet these interactions may in the end lead to physical effects, like instabilities. The simplest situation of this sort is encountered if one breaks 
the gauge group to a non-abelian discrete group. The low energy description of such a models is referred to as a discrete gauge theory. The defects are "magnetic" fluxes which however carry non-abelian quantum numbers [19]. In a two dimensional setting these would give rise to non-abelian anyons. Another class of models is obtained if the unbroken group has several discrete components as well as some non-trivial first homotopy group. In such situations there are monopoles as well as topological fluxes and this may lead to remarkable physical properties. The simplest example of this sort is Alice electrodynamics introduced by A.S. Schwartz in 1982 [20]. The original model is just the Yang-Mills-Higgs system (3), with the Higgsfield $\Phi$ in the five dimensional, symmetric tensor representation of $S U(2)$. The potential is given by:

$$
V=-\frac{1}{2} \mu^{2} \operatorname{Tr}\left(\Phi^{2}\right)-\frac{1}{3} \gamma \operatorname{Tr}\left(\Phi^{3}\right)+\frac{1}{4} \lambda\left(\operatorname{Tr}\left(\Phi^{2}\right)\right)^{2} \quad .
$$

and has three parameters. By a suitable choice of parameters the Higgs field will acquire a vacuum expectation value, $\Phi_{0}$ of the form $\Phi_{0}=\operatorname{diag}(-f,-f, 2 f)$. It follows that the residual gauge group $H=U(1) \ltimes \mathbb{Z}_{2} \sim O(2)$, so, in a certain sense it is the most minimal non-abelian extension of ordinary electrodynamics. The nontrivial $\mathbb{Z}_{2}$ transformation reverses the direction of the electric and magnetic fields and the sign of the charges.

$$
X Q X^{-1}=-Q
$$

with $X$ the nontrivial element of $\mathbb{Z}_{2}$ and $Q$ the generator of the $U(1)$. The generator of $U(1)$ and the nontrivial element of the $\mathbb{Z}_{2}$ do not commute with each other, in fact they anti-commute. This means that the $\mathbb{Z}_{2}$ part of the gauge group acts as a (local) charge conjugation on the $U(1)$ part of the gauge group. Indeed, it is a version of electrodynamics in which charge conjugation symmetry is gauged. From the structure of the (residual) gauge group it is clear what the possible topological defects in this theory are. As $\Pi_{0}\left(U(1) \ltimes \mathbb{Z}_{2}\right)=\mathbb{Z}_{2}$ there will be a topological $\mathbb{Z}_{2}$ flux, denoted as Alice flux, and furthermore as $\Pi_{1}\left(U(1) \ltimes \mathbb{Z}_{2}\right)=|\mathbb{Z}|$ there are also magnetic monopoles in this theory (like in compact ED). The element of the unbroken gauge group associated with the Alice flux contains the nontrivial element of the $\mathbb{Z}_{2}$ part of the gauge group, $X$. This means that if a charge is moved around an Alice flux it gets charge conjugated. At first this might not be such a very interesting observation as charge conjugation is part of the local gauge symmetry of the model. However as mentioned before there is the notion of a relative sign, which is path dependent in the presence of Alice fluxes. This means that if one starts with two equal charges (repulsion) and one moves one of the charges around an Alice flux one ends up with two charges of the opposite sign (attraction), due to the noncommutativity of $X$ and $Q$. This allows for the rather interesting sequence of configurations depicted in Fig 4 where a charge is pulled through a ring of Alice flux. Global charge conservation requires that it leaves behind a (doubly) oppositely charged Alice ring, but charged in a peculiar non-localizable way. The net charge in the region around the ring is nonzero, yet a small test charge 


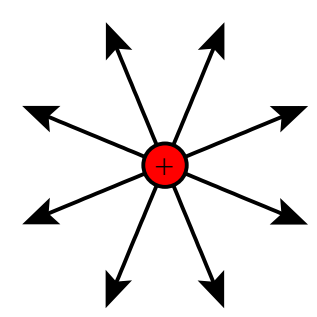

(a)

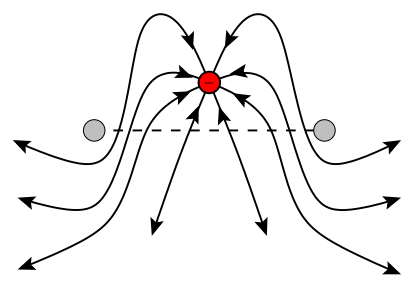

(c)

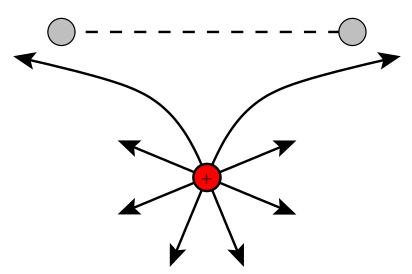

(b)

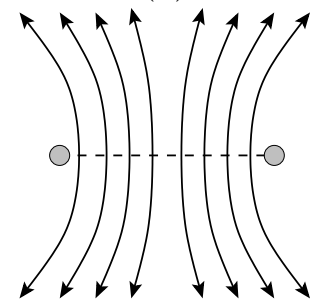

(d)

Figure 4: This sequence of pictures makes clear that the Cheshire phenomenon is generic in these models and does not depend on the particular symmetry of the configuration. Using the fact that due to charge conservation and/or quantization electric field lines cannot cross an Alice flux one is lead to the notion of Cheshire charge.

would be pulled through the ring without encountering any source and then be pushed away on the other side! The surface where the electric field lines change sign is a gauge artefact, a fictitious Dirac sheet bounded by the Alice flux ring and therefore does not carry real charge. Nevertheless, enclosing the whole ring in a closed surface one measures a total net charge. The possibility of this type of non-localizable charge was first noted by Schwarz 20 and is referred to as Cheshire charge 21], referring to the cat in Alice in Wonderland that disappears but leaves his grin behind. One may show that - not surprisingly this Cheshire property also holds for magnetic charges 22 . This is most directly illustrated by an allowed deformation of the core topology of the monopole in this theory. This is shown schematically in Fig 5 We see that because of the director property (i.e. double arrowed nature) of the order parameter field $\Phi_{0}$ it is possible to drill a hole through the core maintaining continuity of the order parameter 23]. The magnetic field lines stay attached to the order parameter and spread out over the minimal surface spanned by the ring, pretty much like ordinary magnetic flux lines would spread when kept together by a super conducting ring. Because of the presence of the additional parameter $\gamma$ in the potential (21), one can imagine that the allowed deformation could lead to a dynamical core instability of the 't Hooft-Polyakov monopole in this theory. It has been shown that this is indeed the case; for a certain range of parameters the magnetic Cheshire configuration has the lowest energy [24]. 


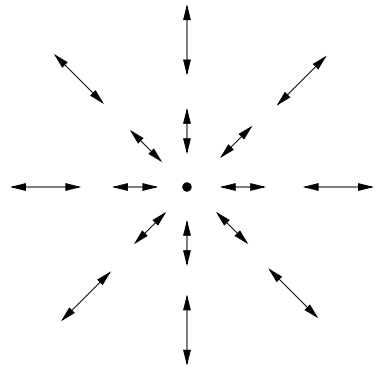

(a)

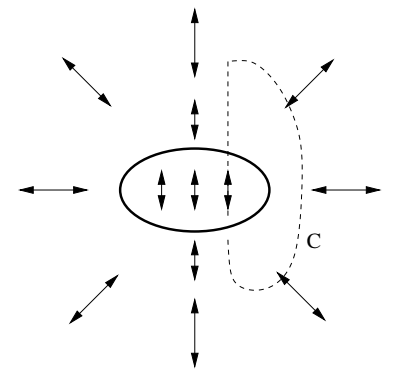

(b)

Figure 5: This figure illustrates the possibility of a smooth deformation of a monopole core topology of a point (a) to magnetically charged Alice ring configuration (b).

\section{$5 \quad$ Physics from moduli space}

The moduli space is basically the space of solutions $\mathcal{M}=\{\mathbf{A}, \mathbf{\Phi}\}$ modulo gauge transformations. This space of physically inequivalent configurations has of course many disconnected components $\mathcal{M}_{m}$ labelled by the topological charge $m$. This classically degenerate moduli space can be described by collective coordinates, which upon quantisation give a semiclassical spectrum of quantum states to which the collection classical monopole solutions give rise. The fact that monopoles can be located anywhere in real space leads to translational zero modes. Modes associated with the compact internal symmetry group will give rise to the "electric" gauge charges that can be implemented in the various topological sectors. The dimensionality of the moduli space in a given topological sector can be computed by calculating the appropriate index for the Dirac type operator in a background gauge. For the $S U(2)$ Bogomol'nyi equations E. Weinberg [25] constructed the operator and used an index theorem of Callias 26] to calculate the dimension of $\mathcal{M}_{m}$, the dimension of the moduli space (i.e. the number of $L^{2}$ normalizable, independent zeromodes) in magnetic sector $m$ with the result

$$
\operatorname{dim} \mathcal{M}_{m}=4|m| \text {. }
$$

Roughly speaking this dimensionality can indeed be interpreted as the number of degrees of freedom of $|m|$ individual fundamental monopoles - each with three translational and one charge degree of freedom. The $m=1$ moduli space has four parameters and the geometry is simply $\mathcal{M}_{1}=\mathbb{R}^{3} \times S^{1}$. The corresponding modes were constructed explicitly by Mottola 27.

In the two-monopole moduli space one may go one step further and discuss the low energy dynamics of monopoles. The isometric decomposition of this space is

$$
\mathcal{M}_{2}=\mathbb{R}^{3} \times \frac{S^{1} \times \overline{\mathcal{M}}_{2}^{0}}{\mathbb{Z}_{2}}
$$

The space $\overline{\mathcal{M}}_{2}^{0}$ is the double cover of the moduli space of centered 2- monopole 
configurations, a 4-dimensional manifold named after Atiyah and Hitchin, who determined the metric from the infinitesimal field modes, i.e. the innerproduct of tangent vectors of this space [28]. The manifold $\overline{\mathcal{M}}_{2}^{0}$ is hyper-Kähler, and an anti-selfdual Euclidean Einstein space with vanishing scalar curvature. The metric has furthermore an $\mathrm{SO}(3)$ isometry group, and therefore can be written in terms of a radial coordinate $r$ and three left invariant one-forms $\sigma_{i}(i=1,2,3)$ that satisfy the relation $d \sigma_{i}=\epsilon_{i j k} \sigma_{j} \sigma_{k}$. The metric becomes:

$$
d s^{2}=\left(\frac{M_{m}}{2}\right)_{2}\left[f(r)^{2} d r^{2}+a(r)^{2} \sigma_{1}^{2}+b(r)^{2} \sigma_{2}^{2}+c(r)^{2} \sigma_{3}^{2}\right] .
$$

and approaches for large $r$ the Euclidean Taub-Nut metric. In the moduli space approximation the classical scattering of two widely separated monopoles is described by the geodesic motion on this space [29, 30, 31]. One may go one step further and construct a Hamiltonian from the canonical Laplacian on the modulispace to discuss the semi-classical bound states and scattering cross sections of monopoles.

In the case of supersymmetric extensions there may be modes associated with supersymmetries as well, which lead to interesting consequences related with duality. We return to these matters later on.

\subsection{Topologically non-trivial Gauge transformations}

In general one has the infinite dimensional group $\mathcal{G}$ of smooth, time independent gauge transformations associated with the structure group $G$

$$
\mathcal{G} \equiv\left\{g: \mathbb{R}^{3} \rightarrow G\right\}
$$

If the group $G$ is broken to a residual gauge group $H$ then we rather like to consider the group $\mathcal{G}_{r}$ of residual gauge transformations defined as those transformation which leave the asymptotic Higgs field invariant

$$
\mathcal{G}_{r} \equiv\left\{g: \mathbb{R}^{3} \rightarrow G \mid g(\mathbf{r}) \in H \text { when }|\mathbf{r}| \rightarrow \infty\right\}
$$

It is now important to distinguish the subgroup of asymptotically trivial gauge transformations $\mathcal{H}_{\infty}$ defined as the group of residual transformations which tend to the identity element at spatial infinity:

$$
\mathcal{G}_{\infty} \equiv\left\{g: \mathbb{R}^{3} \rightarrow G \mid g(\mathbf{r}) \rightarrow 1 \text { when }|\mathbf{r}| \rightarrow \infty\right\} .
$$

Clearly we may consider these transformations as maps from $S^{3}$ to $G$ with the "point at infinity" mapped to the identity element of $G$.

Finally there is the connected component $\mathcal{G}_{\infty}^{0} \subset \mathcal{G}_{\infty}$ of all elements which can be continuously deformed to the identity element $g(\mathbf{r}) \equiv 1$ of $\mathcal{G}_{\infty}$. The group $\mathcal{G}_{\infty}^{0}$ is a normal subgroup of $\mathcal{G}_{\infty}$ consisting of the topologically trivial gauge transformations. In general on has that

$$
\mathcal{G}_{\infty} / \mathcal{G}_{\infty}^{0} \simeq \pi_{3}(G)
$$


saying that the elements of the coset are in one to one correspondence with the homotopy classes of maps $S^{3} \rightarrow G$ with $\infty \mapsto 1$. For abelian $G$ it follows that $\mathcal{G}_{\infty}=\mathcal{G}_{\infty}^{0}$, but for simple Lie groups we have the result that $\pi_{3}(G)=\mathbb{Z}$. and there are nontrivial residual gauge transformations. As will become clear, these topologically nontrivial gauge transformations will be related to the $\theta$ parameter which labels the nontrivial ground states in non-abelian gauge theories.

Imposing Gauss' law on physical states in a given magnetic charge sector means that we require the physical states in that sector to be invariant under $\mathcal{G}_{\infty}^{0}$. The result of the analysis is that there is a group of physical, internal symmetry transformations that generates physically inequivalent configurations and therefore should lead to physical zero modes and will upon quantization be realized on the physical states. It is the group of residual gauge transformations modulo the transformations generated by Gauss' constraint

$$
\mathcal{S} \equiv \mathcal{G}_{r} / \mathcal{G}_{\infty}^{0}
$$

A precise analysis of Balachandran and Giulini 32 33 led to the following structure for the situation for the 't Hooft-Polyakov monopoles in the GeorgiGlashow model.

$$
\mathcal{S}= \begin{cases}\mathbb{Z} \times U(1) & n=0 \\ \mathbb{Z}_{|n|} \times \mathbb{R} & n \neq 0\end{cases}
$$

In the trivial sector this leads to two physical parameters referring to the representation labels of $\mathcal{S}$, but because $\mathcal{S}$ is defined as a quotient, the parameterization of the transformations is not trivial. It should take care of the way the $\theta$ parameter enters in the charged sectors of magnetic monopoles, the socalled Witten effect.

\subsection{The Witten effect: CP violation in the monopole sec- tor}

The Witten effect 34 refers to the shift of the allowed electric charges carried by magnetic monopoles. In other words, the dyonic spectrum of the theory depends on the $\mathrm{CP}$ violating $\theta$ vacuum parameter introduced by 't Hooft. The $\theta$ parameter enters through the addition of a topological term

$$
\mathcal{L}_{\theta}=\frac{\theta e^{2}}{32 \pi^{2}} F \wedge F
$$

to the Lagrangian. This term is a total derivative and therefore does not affect the field equations. However, on the quantum level it does affect the physics and leads to an additional physical parameter in the theory [35, 36, 37, 38].

Witten considered the implementation of the nontrivial internal U(1) transformations on the fields and calculated the Noether charge associated with that symmetry; and found that there is a contribution from the $\theta$ term in the Lagrangian to that $U(1)$ current.

In view of the observations made in the previous subsection we focus on nontrivial gauge transformations which are constant rotations generated by the 
Higgs field (normalized at infinity)

$$
g(\mathbf{r})=\exp [\alpha \Phi(\mathbf{r}) / f] \quad \text { with } \quad g_{\infty}(\hat{\mathbf{r}})=\exp \left[\alpha \hat{\Phi}_{\infty}(\hat{\mathbf{r}})\right]
$$

Applying this transformation gives the infinitesimal changes in the fields

$$
\left\{\begin{array}{l}
\mathbf{a}=-\frac{1}{e f} \mathbf{D} \Phi \\
\phi=0
\end{array}\right.
$$

Now we may calculate the corresponding charge generator $N$ from Noethers theorem:

$$
N=\int d^{3} \mathbf{r}\left[\frac{\partial \mathcal{L}}{\partial\left(\partial_{0} \mathbf{A}\right)} \cdot \mathbf{a}+\frac{\partial \mathcal{L}}{\partial\left(\partial_{0} \Phi\right)} \phi\right]
$$

Substituting the gauge transformations one obtains

$$
N=\int d^{3} \mathbf{r}\left[\left(\mathbf{E}-\frac{\theta e^{2}}{8 \pi^{2}} \mathbf{B}\right) \cdot \mathbf{D} \Phi\right]=\frac{1}{e}\left(q-\frac{\theta e^{2}}{8 \pi^{2}} g\right)
$$

this operator has an integer spectrum, from which the allowed charges in the topological sector $m$ follow

$$
q=\left(n+\frac{\theta m}{2 \pi}\right) e
$$

The electric charges in the dyonic sectors are shifted in a way consistent with the $2 \pi$ periodicity of $\theta$. The shift does not violate the quantization condition for dyons, $q_{1} g_{2}-q_{2} g_{1}=2 \pi n$, because the $\theta$ dependent terms cancel. We have indicated the $\theta$-dependent shift in the electric-magnetic charge lattice (for $\mathrm{SU}(2))$ in Fig 6

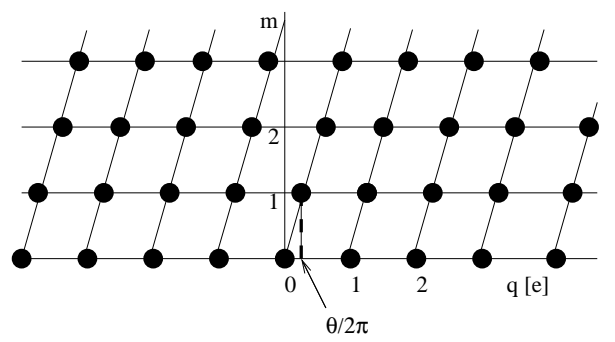

Figure 6: The figure shows the shift of electric charges in the magnetic sectors, due to the $\mathrm{CP}$ violating $\theta$ angle.

An interesting consequence of this shift in the electric charge proportional to theta and the magnetic charge, is the effect of oblique confinement[]. If on considers the vacuum of the unbroken gauge theory as a magnetic superconductor in which the 't Hooft-Polyakov monopoles are condensed, one may wonder what would happen to the condensate if one increases theta. Clearly the total Coulombic repulsion of the monopoles in the vacuum will increase, and 
moreover, if theta reaches $\pi$ the dyon with smallest negative charge will have a smaller total charge and therefore allow for a new ground state with lower energy. In other words, this reasoning suggests a phase transition for $\theta=\pi$ and shows what the physical nature of the transition is. In principle one could imagine also other points on the lattice to condense leading to a variety of oblique confining phases 39$]$.

\subsection{Isodoublet modes: Spin from Isospin}

So far we have looked at the Georgi-Glashow model without introducing additional matter multiplets. It is interesting to do so and to consider how an additional scalar or spinor doublet in the theory affects the moduli space.

We have alluded before to the the spherically symmetric solutions. These where $S O(3)$ symmetric with respect to a generator $\mathbf{J}=\mathbf{L}+\mathbf{T}$ that simultaneously generates space rotations and rigid gauge transformations. In studying the modes for matter fields that couple to the monopole one finds that it is $\mathbf{J}$ that generates the "true" angular momentum, i.e. it is the operator that commutes with the Hamiltonian of the system in the monopole background. This is reminiscent of the situation in abelian monopole physics where the electromagnetic field of a dyonic system of a pole of strength $g$ and a charge $q$ carries an angular momentum $q g / 4 \pi$ (independent of the separation) in a direction pointing from the charge to the pole. One finds that for a charge-pole pair satisfying the minimal Dirac condition $q g=2 \pi$ the possibility of half integer "intrinsic" angular momentum arises. This possibility is also naturally present in the model we have been discussing where the 't Hooft-Polyakov monopole was doubly charged, i.e. $e g=4 \pi$, this is indeed consistent with the presence of doublet fields which have electric charges $q= \pm e / 2$ yielding the minimal value $q g=2 \pi$. Detailed calculations from 't Hooft and Hasenfratz [0] and Jackiw and Rebbi 41] showed that for the scalar doublet modes the generator of the angular momentum does indeed have half integral eigenvalues. Adding to the Lagrangian (3) the term for a doublet $U$

$$
\mathcal{L}_{d}=\left|D_{\mu} U\right|^{2}
$$

with $D_{\mu} U=\left(\partial_{\mu}+i e A_{\mu}^{a} \tau^{a} / 2\right) U$. The classical doublet mode can be written like

$$
U=u(r) \exp \left(-i \alpha_{a} \tau^{a} / 2\right) \mathbf{s}
$$

with three parameters arbitrary parameters $\alpha^{a}$ and $\mathbf{s}$ some constant spinor . The contribution of $\mathcal{L}_{d}$ to the angular momentum generator is given by

$$
\mathbf{J}=-\int d^{3} r \mathbf{r} \times\left[\Pi_{U}^{\dagger}\left(\nabla+i e \mathbf{A}^{a} \tau^{a} / 2\right) U+h . c .\right]
$$

where the conjugate momentum to $\mathrm{U}$ is $\Pi_{U}=D_{0} U$. Making the collective coordinates $\alpha$ in the solution time dependent, i.e. $\alpha=\alpha(t)$ one obtains that $\Pi_{U}^{\dagger}=D_{0} U^{\dagger}=\dot{U}^{\dagger}$ yielding

$$
\mathbf{J}=-\int d^{3} r \dot{U}^{\dagger}(-\mathbf{r} \times \nabla+i \tau / 2) U+\text { h.c. }
$$


where we have used the background monopole solution. With the spherical symmetry of the mode expression (39) for $U$ one is left with the last term in the bracket. So the physical angular momentum $\mathbf{J}$ becomes equal to $\mathbf{T}=\tau / \mathbf{2}$, the generator of the internal transformation, which for the doublet is a (iso)spin one-half representation, i.e. $s=q g / 4 \pi=1 / 2$. And, as advertised, isospin has turned into spin.

\subsection{Fermions from bosons}

If we succeeded in converting half-integral isospin to half-integral spin we should also ask whether we have at the same time constructed fermions out of bosons. In other words, we have to consider the interchange properties of two dyonic composites. Let us rephrase an ingenious argument originally due to Goldhaber [42]. The argument runs as follows. One considers two dyonic composites with electric charge $e$ and magnetic charge $g$ with coordinates $\mathbf{r}_{\mathbf{1}}$ and $\mathbf{r}_{2}$. The corresponding two particle Schrödinger problem, can be separated in a center of mass and a relative coordinate $\mathbf{r}=\mathbf{r}_{1}-\mathbf{r}_{\mathbf{2}}$. The part of the wave function depending on the relative coordinate is of course defined on the space of this relative coordinate. But as the particles are considered to be indistinguishable we may identify the points $\mathbf{r}$ and $-\mathbf{r}$. We furthermore keep the dyons well separated so that the interiors do not take part in the interchange, which means that we exclude the point $\mathbf{r}=0$. The resulting two particle space (taking $r$ fixed) is then topologically equivalent to a two-sphere with opposite points identified i.e. two-dimensional real projective space. A physical interchange corresponds to a closed path in this projective space, and since the first homotopy group $\pi_{1}\left(P \mathbb{R}_{2}\right)=\mathbb{Z}_{2}$ there are two inequivalent classes of wave functions on this space which correspond to the different representations of this $\mathbb{Z}_{2}$. This is the topological origin of the quantum mechanical exchange properties of particles. If there were no electromagnetic field present, the arguments allows one to introduce fermions and bosons as the particles to start of with. In our case these are chosen to be bosons. The electromagnetic interaction term with charge monopole interactions included will take the following form

$$
\mathbf{p}+i e[\mathbf{A}(\mathbf{r})-\mathbf{A}(-\mathbf{r})] .
$$

The effective gauge potential in brackets has zero magnetic field because one subtracts the field in the point $\mathbf{r}$ with the field in the point $-\mathbf{r}$ and these are equal up to a (topologically nontrivial) gauge transformation ${ }^{4}$. Indeed the overall electromagnetic interaction between two particles that carry electric and magnetic charges with the same ratio, are strictly dual to two purely electric charges which have only Coulombic interactions. So there is no net magnetic field in the configuration and the total expression between the brackets must indeed be pure gauge. This implies that by taking the relative particle coordinate around a closed loop (i.e. interchanging the two particles) only two things may happen to the phase of the wave function; because the phase cannot dependent

\footnotetext{
${ }^{4}$ Take for example the potentials as in Eqn 1 then it is clear that $\mathbf{a}_{\hat{n}}(-\mathbf{r})=\mathbf{a}_{-\hat{n}}(\mathbf{r})$
} 
on continuous deformations of the path taken, it can only depend on the homotopy class of the path. In particular since the space is doubly connected the resulting gauge connection may only induce a nontrivial $\mathbb{Z}_{2}$ phase if one goes to the opposite point on the sphere, i.e takes a non-contractible loop. This is indeed the case. In the original non-trivial $U(1)$ monopole bundle one needs two overlapping patches, say northern and southern hemispheres, The gauge potentials in the different patches differ by a gauge transformation

$$
e \mathbf{A}_{I I}=e \mathbf{A}_{I}+\nabla \chi
$$

with the single valuedness condition in the overlap yielding $\chi(\varphi+2 \pi)-\chi(\varphi)=$ $2 \pi m=e g$. The two-particle wave functions are sections of a (non)trivial $\mathbb{Z}_{2}$ bundle over $P \mathbb{R}_{2}$ with transition function $\exp (i e g / 2)=(-1)^{2 s}$. The spin-statistics connection is saved from a painful demise, and we succeeded in making fermions out of bosons.

\subsection{The Rubakov-Callan effect: monopole induced catalysis of baryon number viola- tion}

If a charge moves straight towards a monopole no force is exerted on it and therefore it would just move straight through the monopole. But if this is what happens, the total angular momentum $J$ would not be conserved because in this process $L=0$ and the other term egr $/ 4 \pi$ would change sign. This contradiction is resolved in more realistic quantum mechanical descriptions. For example if we consider a Dirac field in the abelian, minimal $(e g / 4 \pi=1 / 2)$ monopole background we find that in the lowest angular momentum state the radial component of the spin has to satisfy 43 ,

$$
\sigma \cdot \hat{\mathbf{r}}=\frac{e g}{|e g|},
$$

and hence the helicity operator $h$ is related to the charge and equals

$$
h=-\frac{e g}{|e g|} \hat{p}_{r} .
$$

This implies that scattering of a fermion by a monopole in the abelian theory always induces a helicity flip. Though naively the helicity operator commutes with the Hamiltonian and therefore should be conserved, the fact is that careful analysis of the self-adjointness property of the Hamiltonian, leads to boundary conditions for which the helicity operator is not hermitean and therefore helicity needs no longer to be conserved 44 .

In the case of the 't Hooft-Polyakov monopole however, the situation turns out to be radically different. Now one has to return to the analysis of a spinor iso-doublet field in the monopole background. The scattering solutions 45] in the $J=0$ channel describe a process where charge exchange is the mechanism by which the angular momentum conservation is saved. The cross-section is 
basically determined by kinematics and will be of order $\pi / k^{2}$. One may employ the same analysis to the fundamental $S U(5)$ monopole to find out that baryon number would not be conserved in such a process. The minimal monopole typically couples to doublets $\left(d_{3}, e^{+}\right)$and $\left(u_{1}^{c}, u_{2}\right)$ (where the numerical indices refer to color), so in general one obtains processes like:

$$
\begin{aligned}
d_{3 R}+\text { dyon } & \rightarrow e_{R}^{+}+d y o n \\
e_{R}^{+}+d y o n & \rightarrow d_{3 l}+d y o n \\
u_{1 R}^{c}+d y o n & \rightarrow u_{2 R}+d y o n \\
u_{2 L}+\text { dyon } & \rightarrow u_{1 L}^{c}+d y o n
\end{aligned}
$$

In this mode analysis also electric charge and color conservation appear to be violated, which is of course an artifact because the back reaction of the monopole is not taken care of. In a full quantum mechanical treatment one finds that the charge degrees of freedom on the monopole will be excited as to ensure conservation of the charges related to local symmetries. But the violation of baryon and lepton number remains. Rubakov and Callan took the previous analysis some dramatic steps further. The studied the full quantum dynamics in the spherically symmetric $J=0$ channel, in which the fermion monopole system reduces to a two-dimensional chiral Schwinger model on a halfline. This model has been analyzed in detail in the fermionic formulation by Rubakov [46, 47] and by Callan 48, 49] after bosonisation. Most remarkable is the persistence of the large geometric cross section which is not cutoff by the symmetry breaking scale, therefore baryons can decay with dramatic rates at low energy in the presence of grand unified monopoles.

\section{Supersymmetric monopoles}

The study of monopoles has become a crucial ingredient in understanding the physical properties of non-abelian gauge theories. Remarkable progress has been made in particular in the realm of suppersymmetric gauge theories where a number of important exact results have been obtained. In the following subsections we review some of the turning points along these lines of development. To fix the setting, let us consider the $N=2$ supersymmetric $S U(2)$ Yang Mills theory with Lagrangian

$$
\mathcal{L}=-\frac{1}{4} F^{2}+\bar{\Psi} \not D \Psi+\frac{1}{2}(D \phi)^{2}+\frac{1}{2}(D \chi)^{2}-e \bar{\Psi}\left[\phi+i \gamma_{5} \chi, \Psi\right]+\frac{1}{2} e^{2}[\phi, \chi]^{2}
$$

The theory involves one $\mathrm{N}=2$ chiral (or vector) supermultiplet in the adjoint representation of the gauge group. This multiplet consists of a the gauge field $A_{\mu}$, two Weyl spinors combined in a single Dirac spinor $\Psi$, a scalar $\phi$ and pseudoscalar $\chi$. Alternatively one may choose to keep the two Weyl spinors and combine both scalar components in a single complex scalar field. In this formulation the potential is just given by $\frac{1}{2} e^{2}\left[\Phi, \Phi^{\dagger}\right]^{2}$. Setting the fields $\Psi$ and $\chi$ to zero we recover the bosonic Georgi-Glashow model in the BPS-limit, with 
the well known monopole solution. At this point it is convenient to rescale the whole supermultiplet by the coupling constant $e$, giving an overall factor $1 / e^{2}$ in front of the Lagrangian. satisfy

It is particularly interesting to study the fermionic zero modes that have to

$$
i \not D \Psi-[\phi, \Psi]=0
$$

Solutions can be generated applying a supersymmetry transformation to the classical, bosonic monopole configuration and read of what it yields for the spinor. The transformation rule gives

$$
\delta \Psi=\left(\sigma_{\mu \nu} F^{\mu \nu}-\not D \phi+\gamma_{5}[\chi, \phi]+i \gamma_{5} \not D \chi\right) \varepsilon
$$

Using the Bogomol'nyi equations only one component of the first two terms survives:

$$
\Psi=\left(\begin{array}{c}
\chi^{+} \\
\chi^{-}
\end{array}\right)=\left(\begin{array}{cc}
\sigma \cdot \mathbf{B} & 0 \\
0 & 0
\end{array}\right)\left(\begin{array}{l}
s^{+} \\
s^{-}
\end{array}\right)
$$

and one obtains the solution as first discussed by Jackiw and Rebbi,

$$
\chi^{+}=\sigma \cdot \mathbf{B} s^{+} \text {and } \chi^{-}=0
$$

Here the two-component spinor $s^{+}$is still arbitrary so that we end up with two zeromodes, which are furthermore charge conjugation invariant. These modes of course also exist in non-supersymmetric versions of the model. In the expansion of the quantized Dirac field these modes have to be included, we have to write

$$
\Psi=c_{1} \psi_{1}+c_{2} \psi_{2}+\sum_{p}\left(b_{p} \psi_{p}+d_{p}^{\dagger} \psi_{p}^{c}\right)
$$

The anticommutator of $\Psi$ and $\Psi^{\dagger}$ imposes that the $c$ operators obey a Clifford algebra $\left\{c_{i}, c_{j}^{\dagger}\right\}=\delta_{i j}$. This algebra has a 4 -dimensional representation with states that have the properties indicated in the following table:

\begin{tabular}{|l|c|c|c|c|}
\hline State & $\mid++>$ & $\mid+->$ & $-+>$ & $-->$ \\
\hline Fermion number & 1 & 0 & 0 & -1 \\
\hline Spin & 0 & \multicolumn{2}{|c|}{$\frac{1}{2}$} & 0 \\
\hline
\end{tabular}

The 4-fold ground state degeneracy of the $N=2$ supersymmetric monopole

The conclusion is thus that the groundstate of the $\mathrm{N}=2$ supersymmetric monopole is 4 -fold degenerate.

\subsection{The supersymmetry algebra with central charge}

Witten and Olive 50 studied the way monopoles behave in supersymmetric gauge theories from a different angle. An immediate motivation is the fact that the 
BPS limit is implied by supersymmetry. By studying the super-algebra and its representations they obtained the remarkable result that states saturating the Bogomol'nyi bound remain doing so on the quantum level. The reason is that the BPS states have to form special so-called short representations of the algebra.

The $\mathrm{N}=2$ algebra of super charges allows for central charges $U$ and $V$ and has the following general form:

$$
\left\{Q_{\alpha i}, \bar{Q}_{\beta j}\right\}=\delta_{i j} \gamma^{m} u_{\alpha \beta} P_{\mu}+\varepsilon_{i j}\left(\delta_{\alpha \beta} U+\left(\gamma_{5}\right)_{\alpha \beta} V\right)
$$

The two charges $Q_{\alpha}$ are two-component Majorana spinors. In the monopole state one obtains that the central charge $V$ is nonvanishing

$$
V=\int d^{3} x[\phi(\nabla \cdot \mathbf{B})+\chi(\nabla \cdot \mathbf{E})]=g f \geq 0
$$

$U$ is obtained by interchanging $\Phi$ and $\chi$ and equals zero in the pure monopole case. Evaluating the algebra in the rest frame gives

$$
\left\{Q_{\alpha i}, \bar{Q}_{\beta j}\right\}=\delta_{i j} \delta_{\alpha \beta} M+\varepsilon_{i j}\left(\gamma_{5} \gamma_{0}\right)_{\alpha \beta} \quad g f
$$

Positivity of the anticommutator leads to the bound $M^{2} \geq(g f)^{2}$. Something special happens if the bound is saturated, then the representation theory of the $\mathrm{N}=2$ algebra alters and allows for a so called short representation of $2^{N}=4$ states, with spin content $\left(\frac{1}{2}, 0^{+}, 0^{-}\right)$. Normal, "long" representations have $2^{2 N}$ states. We see that the zeromodes discussed in the previous section indeed form a short representation. And what about possible excited dyonic bound states one might wonder. These do exist and have been analyzed [51] and give - as expected - rise to long representations because they do not correspond to zeromodes and hence do not satisfy the Bogol'nyi bound. This distinction between short and long representations can be compared to the difference between massive and massless representations of the Lorentz group. In the quantum BPS limit of the $\mathrm{N}=2$ theory we arrive at the conclusion that the monopole groundstate forms a scalar multiplet (containing a spin 1/2 doublet). Osborn extended this analysis to the $N=4$ case obtaining that the lowest monopole states form the short $\mathrm{N}=4$ multiplet now containing 16 states consisting of a vector, four spinors and six scalars, i.e. a magnetic copy of the vector multiplets that define the theory 52 .

\subsection{Duality regained}

In 1979 Montonen and Olive [53] made a daring conjecture concerning spontaneously broken gauge theories. Inspired by the the mass formula for BPS states they conjectured that — in the full quantum theory - there would be a non-abelian version of electric magnetic duality realized in the supersymmetric $S O(3)$ model. This duality would be a four dimensional analogue of the equivalence of the Sine-Gordon Theory and the massive Thirring model in two dimensions. The strongest form of it would be realized in the $\mathrm{N}=4$ theory. In 
this case the quantum numbers of the electric and magnetic BPS states are such that the model could be self-dual in the sense that the massive vector bosons would be mapped on the monopoles and vice versa, implying that the charges get also mapped onto each other, i.e. $e \leftrightarrow g=4 \pi / e$. This is clearly a strongweak coupling duality in that the fine structure constant would be mapped to its inverse. The conjecture amounts to the statement that in the strong coupling regime the physics would be described by a weakly interacting spontaneously broken "magnetic" gauge theory with a dual gauge group $\tilde{G} \simeq S O(3)$. There appears considerable evidence for this idea:

- The BPS mass relation $m=f \sqrt{q^{2}+g^{2}}$.

- The BPS mass formula receives no quantum corrections as it appears in the central charge of the supersymmetry algebra.

- In the model there is no charge renormalization.

- The spin content of the electric and magnetic representations is the same (1 vector, 4 spinors and 6 (pseudo)scalars).

This bold idea has in recent years developed into a broad research field mainly due to the work of Seiberg and Witten on electric-magnetic dualities in general supersymmetric gauge theories. In the following sections we briefly summarize some aspects of this work.

\section{3 $\mathrm{SL}(2, \mathbb{Z})$ duality}

We may extend the Montonen-Olive duality to a full $S L(2, \mathbb{Z})$ duality by including also the $\theta$ parameter. Let us introduce the complex coupling parameter

$$
\tau \equiv \frac{\theta}{2 \pi}+\imath \frac{4 \pi}{e^{2}}
$$

We recall formula that for the dyons with magnetic charge $g=4 \pi m / e$ in the presence of the $\theta$ parameter the electric charges are given by $q=n e+m \theta e / 2 \pi$. The exact BPS mass formula as it follows from the central charge of supersymmetry algebra can now be casted in the form

$$
M(m, n)=e f|n+m \tau| \quad m, n \in \mathbb{Z}
$$

This BPS mass formula is invariant under $S L(2, \mathbb{Z})$ transformations which act as follows. Let $M$ be an element of $S L(2, \mathbb{Z})$, which is defined as

$$
M=\left(\begin{array}{cc}
a & b \\
c & d
\end{array}\right), \quad \text { with } a, b, c, d \in \mathbb{Z} \text { and } a d-b c=1,
$$

then the parameter $\tau$ transforms as

$$
\tau \rightarrow \tau \prime=\frac{a \tau+b}{c \tau+d}
$$


whereas the BPS states transform as

$$
(m, n) \rightarrow\left(m^{\prime}, n^{\prime}\right)=(m, n) M^{-1}=(m, n)\left(\begin{array}{cc}
d & -b \\
-c & a
\end{array}\right) .
$$

The group $S L(2, \mathbb{Z})$ is generated by $T: \tau \rightarrow \tau+1$ and $S=\tau \rightarrow-1 / \tau$. The generator $T$ generates a shift of $\theta$ over $2 \pi$, while for $\theta=0$ the $S$ generator corresponds to the original Montonen-Olive duality transformation, with $e \rightarrow$ $4 \pi / e$ and $(m, n) \rightarrow(-n, m)$. The $S L(2, \mathbb{Z})$-duality conjecture reads that a theory would be invariant under the $S L(2, \mathbb{Z})$ transformations on the dyonic spectrum of BPS states.

From the mass formula one may deduce a stability condition. One easily sees that the following inequality between different mass states holds

$$
M\left(m_{1}+m_{2}, n_{1}+n_{2}\right) \leq M\left(m_{1}, n_{1}\right)+M\left(m_{2}, n_{2}\right)
$$

implying that the strict equality holds if and only if $m_{1}+m_{2}$ and $n_{1}+n_{2}$ are relative prime. A dyon with magnetic and electric quantum numbers which are relative prime should therefore be stable, though as we will see later on, the stability depends on which extended supersymmetry is realized.

\subsection{The Seiberg-Witten results: the $\mathrm{N}=2$ vacuum struc- ture}

The question what happens to electric-magnetic duality for $\mathrm{N}=2$ or $\mathrm{N}=1$ supersymmetric gauge theories was answered by some remarkable exact results obtained by Seiberg and Witten [54, 55. The N=2 theory is of course asymptotically free, allowing a choice of scale. The question is to understand the vacuum structure of the theory and the dependence of the BPS spectrum on the vacuum parameters. The classical potential is given by

$$
V(\Phi)=\frac{1}{e^{2}}\left[\Phi, \Phi^{\dagger}\right]^{2},
$$

consequently there is a continuous family of classical vacua because for the potential to acquire its minimum it suffices for $\Phi$ and $\Phi^{\dagger}$ to commute. There is a global $U(1) \mathrm{R}$ symmetry of the classical potential on the classical level which gets broken by instanton effects to a global $\mathbb{Z}_{8}$. The scalar field is doubly charged under this discrete group. A constant value $\Phi=a \tau_{3} / 2$ breaks the gauge group to a $U(1)$. A gauge invariant parameterization of the classical vacua is given by the complex parameter

$$
u=\frac{1}{2} a^{2}=\operatorname{Tr} \Phi^{2}
$$

Because $u$ has charge $k=4$ under the $\mathbb{Z}_{8}$ group, this symmetry gets broken to a global $\mathbb{Z}_{2}$ which acts on the moduli space as $u \rightarrow-u$. On a classical level there is only one special point, the point $u=0$ where the full $S U(2)$ symmetry is restored. 
The quantum moduli space parameterized by the the vacuum expectation value $u=<\operatorname{Tr} \Phi^{2}>$ depends on the effective action, which in turn depends on a single holomorphic function $\mathcal{F}(a)$. Witten and Seiberg succeeded in explicitly calculating $a(u)$ and $a_{D} \equiv(\partial \mathcal{F} / \partial a)(u)$ in terms of certain hypergeometric functions from the holomorphicity constraint combined with certain information on the possible singularities and some (one loop) perturbative results. This information is crucial because it determines the masses of the BPS states as a function of $u$ again from the central charge in the $\mathrm{N}=2$ supersymmetry algebra as

$$
M(m, n)=\sqrt{2}\left|n a(u)-m a_{D}(u)\right|
$$

We see that classically $\tau_{c l}=a_{D} / a$, however on the quantum level $\tau$ is rather defined as $\tau=\left(\partial^{2} \mathcal{F} / \partial a^{2}\right)(u)$. The parameters $a$ and $a_{D}$ are both complex and generically the masses can be represented on a lattice where the mass just corresponds to the Euclidean length of the corresponding lattice vector $(m, n)$. The situation is similar to the one we described before where the triangle inequality determines the stability of various states in the lattice. The interpretation of the phase structure and the BPS spectrum depends critically on the singularities of $a$ and $a_{D}$ in the $u$ plane. There are branchpoints for $u= \pm 1$ and $u=\infty$ with cuts along the (negative) real axis for $a_{D}$ extending to $u=-1$ and for $a$ to $u=1$. We see that the singularities have moved away from the classical point $u=0$. From the singularity structure one may obtain the monodromies generated on the vector $\left(a, a_{D}\right)$ by going around the singularities. The requirement that the physical spectrum should be invariant under the action of the monodromy group will put constraints on which states are allowed.

Of particular interest are the points in the $u$ plane where $a_{D} / a \in \mathbb{R}$ because then the lattice degenerates and instabilities as well as massless states may develop. This situation arises for a locus of points which form a closed curve $\mathcal{C}$, called the curve of marginal stability. We have depicted the situation in Fig[7] On $\mathcal{C}$ the ratio $a_{D} / a$ takes on all values in the interval $[-1,1]$. The large $u$ region is the weakly coupled semiclassical region whereas in the interior domain near and inside the curve of marginal stability, we are dealing with the strong coupling regime. It is clear that we have to distinguish three different regions, the region outside the curve $R_{\text {out }}$, and inside the curve the regions above and below the real axis, $R_{i n}^{ \pm}$. The interpretation of the curve is as follows. Clearly as long as we do not cross it the spectrum will depend smoothly on $u$ and cannot develop any instabilities, neither inside, nor outside the curve. It follows from the mass formula (64) that on the curve massless states may develop. For $M=0$ we see that we must have $n / m=a_{D} / a \in[-1,1]$. In fact naively, that means semi-classically, one would expect that any state with $n / m \in$ $[-1,1]$ will become massless exactly at the corresponding point on $\mathcal{C}$ where $a_{D} / a(u)=n / m$. One clearly expects that the effective action and therefore $a$ and $a_{D}$ would develop singularities, but this - as followed from the result of Seiberg and Witten - only happens for $u= \pm 1$. Conversely if a massless state would develop for some point $u$ in the moduli space it would give rise to a singularity but there are no other singularities than the ones just mentioned, 


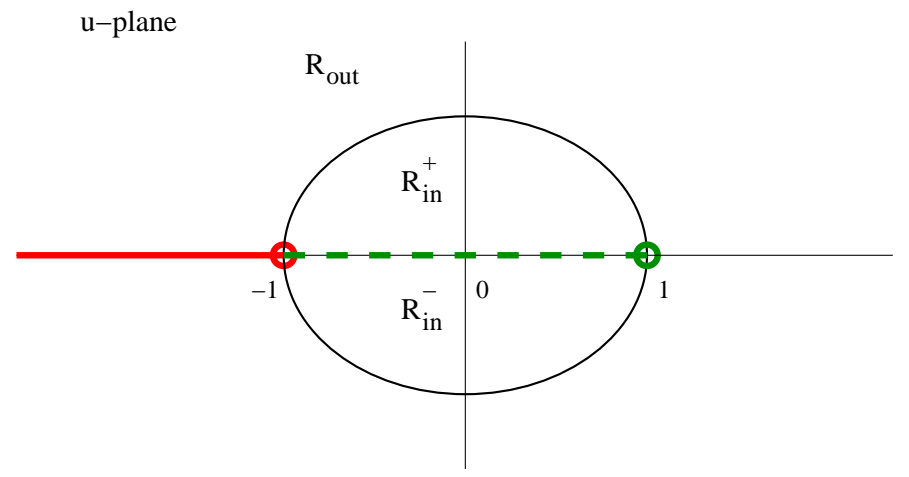

Figure 7: This figure shows the singularity structure of $a(u)$ and $a_{D}(u)$. On the curve the quotient $a_{D} / a$ becomes real, massless states only occur at $u= \pm 1$.

which leads us to conclude that such states should be excluded. The point $\mathrm{u}=+1$ corresponds to a massless state $(0, \pm 1)$ a monopole or anti-monopole, whereas the point $u=-1$ corresponds to the (anti-)dyons $\pm( \pm 1,1)$. These points correspond to groundstates where monopoles/dyons become massless and hence will condense to realize the confining phases anticipated by Mandelstam and 't Hooft in the seventies. A striking result. Adding to this the monodromy constraints mentioned before, the result for the $N=2$ theory without extra matter multiplets can be summarized as follows. In the weak coupling regime the spectrum consists only of the states $\{ \pm(1,0), \pm(n, 1) ; n \in \mathbb{Z}\}$. In the strong regime similar arguments [56, 57] yield that the spectrum is further reduced to just states that become massless $\{ \pm(01), \pm(-1,1)\}$ for the region above and $\{ \pm(01), \pm(-1,1)\}$ for the region below the real axis.

\section{Beyond $S U(2)$ : gauge groups and integrable systems}

The construction of general multi-monopole monopole solutions to the Bogomol'nyi equations for a theory with some general gauge group has been a source of inspiration for a full generation of mathematical physicists. Substantial progress has been made in constructing explicit solutions, and many intriguing relations have been found with various classes of integrable systems in lower dimensions. From these analogies, various solution techniques for (multi)monopoles have been developed especially if additional symmetries were imposed, lowering the effective spacetime dimensionality of the system. This in a first wave gave rise to obtaining multi-monopole solutions for gauge group $\mathrm{SU}(2)$, for example by exploiting Bcklund transformation techniques [58, 59]. An general framework to discuss the selfduality and Bogomol'nyi equations based on algebraic geometry and the use of twistors was introduced by Ward 60, 61] 
and Atiyah and Ward $[2]$. Another general approach, based on the theory complex vector bundles, developed to solve the general instanton problem, usually referred to as the Atiyah-Hitchin-Drinfeld-Manin construction 63, was extended by Nahm for the general Bogomol'nyi equations (see below).

\subsection{Spherical symmetry: Toda systems}

The simplest class of monopoles for which the exact solutions were constructed is the class of spherically symmetric solutions, where one defines a generalized $S O$ (3) generator $\mathbf{J}=\mathbf{L}+\mathbf{T}$ and imposes the condition 9 on the fields. In the present context $\mathbf{T}$ generates some $S U(2)$ subgroup of the the full gauge group. If one chooses the minimal regular embedding corresponding to the (simple) roots of the full gauge algebra one obtains the fundamental monopoles discussed before. The choice of a non-regular embedding leads to more interesting results. For example, the choice of the maximal or better principal $S U(2)$ embedding, where the fundamental (vector) representation branches to a single irreducible $S U(2)$ representation leads to one- (two-) dimensional conformally invariant Toda-like systems 64, 65, 66. The Bogomol'nyi system reduces to

$$
\partial \bar{\partial} \rho_{i}=\sum_{j=1}^{\ell} K_{i j} e^{\rho_{j}},
$$

where we have introduced complex coordinates

$$
z=r+i t, \quad \bar{z}=r-i t .
$$

These finite Toda-like systems are completely characterized by the Cartan matrix $K$ of the Lie algebra of $G$, defined as the (asymmetrically) normalized inner product of the $\ell$ (= rank $G$ ) simple roots $\gamma_{a}$

$$
K_{i j} \equiv 2 \vec{\gamma}_{i} \cdot \vec{\gamma}_{j} /\left(\vec{\gamma}_{j} \cdot \vec{\gamma}_{j}\right)
$$

The $S U(2)$ version of this system is just the Liouville equation initially obtained in this context by Witten 67. The complex equations yield the instanton solutions, the restriction to $\rho_{i}=\rho_{i}(r)$ yields the monopoles. These systems have returned in the context of conformal field theory models for critical systems. The cases for arbitrary $S U(2)$ embeddings involve certain non-abelian generalizations of these finite Toda systems 68 .

\subsection{Axial symmetry: nonlinear sigma models}

Let us briefly consider the axially symmetric case where one imposes symmetry with respect to a single mixed space and gauge rotation. The Bogomol'nyi equations now reduce to an integrable, non-compact, non-linear sigma-model in a curved two-dimensional $(\rho, z)$-space as followed from an analysis of Bais and Sasaki [69];

$$
\nabla\left[\rho(\nabla \mu) \mu^{-1}\right]=0
$$


with $\nabla \equiv\left(\partial_{\rho}, \partial_{z}\right)$. The field variables $\mu$ are defined as follows:

$$
\mu \equiv g^{\dagger} g, \quad g=g(\rho, z) \in G^{*} .
$$

The group $G^{*}$ is any non-compact real form of $G$ based on a symmetric decomposition of the Lie-algebra $G=K+P$, with

$$
[K, K] \subset K \quad, \quad[P, K] \subset K \quad, \quad[P, P] \subset K .
$$

As follows from its definition, $\mu$ is in fact an element of the symmetric noncompact coset space $G^{*} / K$, where $K$ is the maximal compact subgroup of $G^{*}$. For $S U(2)$ the system of Eqn. [68) reduces to the case studied by Manton[70] and to the Ernst equation - well known in general relativity.

\subsection{The Nahm equations.}

We have mentioned in passing that classical Bogomol'nyi or self-dual YangMills equations for monopole respectively instantons differ primarily by their spacetime structure and choice of boundary conditions. This means that the techniques to solve them should be quite similar. The general instanton problem was reduced to a entirely algebraic problem by Atiyah, Hitchin, Drinfeld and Manin 63, the so-called ADHM construction. Nahm adapted this method for the general Bogoml'nyi equations, not surprisingly referred to as the ADHMN construction [71, 72]. Nahm reduced the Bogomol'nyi system to a system of of coupled nonlinear ordinary differential equations for three $(n \times n)$ matrix valued functions $T_{i}(i=1,2,3)$ of some variable $z$ defined on an appropriate interval. The variable $z$ indicates the Fourier components of the time variable $x_{0}$. The equations read:

$$
\frac{d T_{i}}{d z}=i \epsilon_{i j k}\left[T_{j}, T_{k}\right]
$$

The way one arrives at this system of equations starts out with the zero-modes $\psi^{(r)}(x, z)$ of the covariant equation for a Weyl spinor in the fundamental representation of the gauge group in the monopole background. To be definite we assume in the following that the gauge group $G=S U(N)$ [73. The fundamental spinor has to satisfy:

$$
D^{\dagger} \psi(\mathbf{x}, \mathbf{z})=0
$$

with the operator defined as

$$
D=\sigma \cdot(\nabla+i e A)-z+\Phi
$$

The matrices $T_{i}$ are defined as,

$$
T_{i}^{(r s)}(z)=\int \psi^{(r) \dagger}(x, z) x_{i} \psi^{(s)}(x, z) d^{3} x
$$

The indices run $r$ and $s$ run over the number of zero-modes $n=n(z)$ of the adjoint operator $D^{\dagger}$ in the given sector with topological (magnetic) charge $m$. 
Clearly the number of normalizable zero-modes depends on $z$, and consequently the dimensionality of the $T$ matrices does as well. The tricky part sits in the boudary conditions one has to impose on $z$, these depend on the asymptotic behavior of the Higgs field,

$$
\Phi \simeq \sum_{\ell=1}^{N} P_{\ell}\left[z_{\ell}+\frac{k_{\ell}}{2|\mathbf{x}|}\right] \quad|\mathbf{x}| \rightarrow \infty
$$

The $P_{\ell}$ are spherical Legendre functions. In terms of the parameters $z_{\ell}$ and $k_{\ell}$ the number of normalizable modes to the spinor equation turns out to be

$$
n(z)=\sum_{\ell=1}^{N} k_{\ell} \theta\left(z-z_{\ell}\right) .
$$

So $z$ ranges between the largest and smallest eigenvalues of the Higgs field and $n(z)$ jumps at points where $\mathrm{z}$ becomes equal to an eigenvalue of $\Phi$ (except if the eigenvalues happen to be degenerate).

To reconstruct the original gauge and scalar fields given the $T_{i}$ matrix functions, one goes in principle the opposite direction. One first solves the linear equation:

$$
\triangle^{\dagger} v_{k}(z, \mathbf{x})=0
$$

with the $(n \times n)$ quaternionic operator given by

$$
\triangle=i \partial_{z}+(\mathbf{x}+i \mathbf{T}) \cdot \sigma .
$$

It can be shown that this equation has $N$ solutions for $S U(N)$. Setting the normalized solutions $v_{k}$ in a $(n(z) \times N)$ matrix $V$ one may extract the gauge potentials and scalar field which solve the original Bogomol'nyi problem by computing

$$
\begin{aligned}
& \mathbf{A}=\int d z V^{\dagger} \nabla V \\
& \Phi=\int d z z V^{\dagger} V
\end{aligned}
$$

where we have normalized

$$
\int d z V^{\dagger} V=1
$$

The Nahm equations form an integrable system and have popped up in many places most recently in connection with D-brane descriptions of monopoles in M-theory.

\section{Conclusions and outlook}

"Sag mir wo die Blumen sind, wo sind sie geblieben..."

M. Dietrich (1931) 
We have reviewed the physics of magnetic monopoles in non-abelian gauge theories. We have touched upon many different subjects, but could not - in the limited space available - go into much detail. Quite a few subjects were left out, in that sense the particular choices made did not reflect the limited interest of the author but rather his limited expertise. The study of monopoles which received little encouragement from experiment remains a highly interesting topic for theoretical research. To be or not to be remains for the time being, an open question.

Acknowledgement: The author wishes to thank many of his (former) students and colleagues for inspiring collaborations on a wide variety of questions concerning magnetic monopoles.

\section{References}

[1] P.A.M. Dirac. Quantised singularities in the electromagnetic field. Proc.Roy.Soc., A(133):60, 1931.

[2] H. Hopf. Über die Abbildungen der dreidimensionalen Sphäre auf die Kugelfläche. Math. Ann., (104):637, 1931.

[3] G. 't Hooft. Magnetic monopoles in unified gauge theories. Nucl. Phys., B79:276-284, 1974.

[4] A. M. Polyakov. Particle spectrum in quantum field theory. JETP Lett., 20:194-195, 1974.

[5] E. B. Bogomolny. Stability of classical solutions. Sov. J. Nucl. Phys., 24:449, 1976.

[6] M. K. Prasad and C. M. Sommerfield. An exact classical solution for the 't Hooft monopole and the Julia-Zee dyon. Phys. Rev. Lett., 35:760-762, 1975.

[7] F. Englert and P. Windey. Quantization condition for 't Hooft monopoles in compact simple lie groups. Phys. Rev., D14:2728, 1976.

[8] P. Goddard, J. Nuyts, and D. I. Olive. Gauge theories and magnetic charge. Nucl. Phys., B125:1, 1977.

[9] S. R. Coleman. Classical lumps and their quantum descendents. Lectures delivered at Int. School of Subnuclear Physics, Ettore Majorana, Erice, Sicily, Jul 11-31, 1975.

[10] A.S. Shvarts Yu.S. Tyupkin, V.A. Fateev. Topologically nontrivial particles in quantum field theory. Pisma Zh.Eksp.Teor.Fiz., 22:192-194, 1975.

[11] A. S. Schwarz. Magnetic monopoles in gauge theories. Nucl.Phys., B112:358-364, 1976. 
[12] M. I. Monastyrsky and A. M. Perelomov. Some remarks on monopoles in gauge field theories. JETP Lett., 21:43, 1975.

[13] A et al. Balachandran. Topology and the problem of colour. Phys. Rev. Lett, 50:1553, 1983.

[14] P. Nelson and S. R. Coleman. What becomes of global color. Nucl. Phys., B237:1, 1984.

[15] F. A. Bais and B. J. Schroers. Quantization of monopoles with non-abelian magnetic charge. Nucl. Phys., B512:250-294, 1998.

[16] F. A. Bais and R. Laterveer. Exact $Z(N)$ monopole solutions in gauge theories with nonadjoint higgs representations. Nucl. Phys., B307:487, 1988.

[17] F. A. Bais. The topology of monopoles crossing a phase boundary. Phys. Lett., 98B:437, 1981.

[18] R. A. Brandt and F. Neri. Stability analysis for singular nonabelian magnetic monopoles. Nucl. Phys., B161:253-282, 1979.

[19] F. A. Bais. Flux metamorphosis. Nucl. Phys., B170:32, 1980.

[20] A. S. Schwarz. Field theories with no local conservation of the electric charge. Nucl. Phys., B 208:141, 1982.

[21] M. Alford, K. Benson, S. Coleman, J. March-Russell, and F. Wilczek. Zero modes of nonabelian vortices. Nucl. Phys., B 349:414-438, 1991.

[22] M. Bucher, H.-K. Lo, and J. Preskill. Topological approach to Alice electrodynamics. Nucl. Phys., B386:3-26, 1992.

[23] F. A. Bais and P. John. Core deformations of topological defects. Int. J. Mod. Phys., A10:3241-3258, 1995.

[24] F. A. Bais and J. Striet. On a core instability of 't Hooft-Polyakov monopoles. Phys. Lett., B540:319-323, 2002.

[25] E. J. Weinberg. Parameter counting for multi - monopole solutions. Phys. Rev., D20:936-944, 1979.

[26] Constantine Callias. Index theorems on open spaces. Commun. Math. Phys., 62:213-234, 1978.

[27] E. Mottola. Zero modes of the 't Hooft-Polyakov monopole. Phys. Lett., B79:242, 1978.

[28] G. W. Gibbons and N. S. Manton. The moduli space metric for well separated BPS monopoles. Phys. Lett., B356:32-38, 1995.

[29] N. S. Manton. A remark on the scattering of BPS monopoles. Phys. Lett., B110:54-56, 1982. 
[30] M. F. Atiyah and N. J. Hitchin. Low-energy scattering of nonabelian monopoles. Phys. Lett., A107:21-25, 1985.

[31] G. W. Gibbons and N. S. Manton. Classical and quantum dynamics of BPS monopoles. Nucl. Phys., B274:183, 1986.

[32] A. P. Balachandran. Gauge symmetries, topology and quantization. 1992.

[33] D. Giulini. Asymptotic symmetry groups of long ranged gauge configurations. Mod. Phys. Lett., A10:2059-2070, 1995.

[34] E. Witten. Dyons of charge e $\theta / 2 \pi$. Phys. Lett., B86:283-287, 1979.

[35] G. 't Hooft. Symmetry breaking through Bell-Jackiw anomalies. Phys. Rev. Lett., 37:8-11, 1976.

[36] Gerard 't Hooft. Computation of the quantum effects due to a four- dimensional pseudoparticle. Phys. Rev., D14:3432-3450, 1976.

[37] R. Jackiw and C. Rebbi. Vacuum periodicity in a Yang-Mills quantum theory. Phys. Rev. Lett., 37:172-175, 1976.

[38] Jr. Callan, Curtis G., R. F. Dashen, and David J. Gross. The structure of the gauge theory vacuum. Phys. Lett., B63:334-340, 1976.

[39] G. 't Hooft. Topology of the gauge condition and new confinement phases in nonabelian gauge theories. Nucl. Phys., B190:455, 1981.

[40] P. Hasenfratz and G. 't Hooft. A fermion - boson puzzle in a gauge theory. Phys. Rev. Lett., 36:1119, 1976.

[41] R. Jackiw and C. Rebbi. Spin from isospin in a gauge theory. Phys. Rev. Lett., 36:1116, 1976.

[42] A. S. Goldhaber. Spin and statistics connection for charge - monopole composites. Phys. Rev. Lett., 36:1122-1125, 1976.

[43] Yoichi Kazama, Chen Ning Yang, and Alfred S. Goldhaber. Scattering of a Dirac particle with charge $Z e$ by a fixed magnetic monopole. Phys. Rev., D15:2287-2299, 1977.

[44] A. S. Goldhaber. Dirac particle in a magnetic field: Symmetries and their breaking by monopole singularities. Phys. Rev., D16:1815, 1977.

[45] W. J. Marciano and I. J. Muzinich. An exact solution of the Dirac equation in the field of a 't Hooft-Polyakov monopole. Phys. Rev. Lett., 50:1035, 1983.

[46] V. A. Rubakov. Superheavy magnetic monopoles and proton decay. JETP Lett., 33:644-646, 1981. 
[47] V. A. Rubakov. Adler-Bell-Jackiw anomaly and fermion number breaking in the presence of a magnetic monopole. Nucl. Phys., B203:311-348, 1982.

[48] C. G. Callan Jr. Dyon - fermion dynamics. Phys. Rev., D26:2058-2068, 1982.

[49] C. G. Callan Jr. Disappearing dyons. Phys. Rev., D25:2141, 1982.

[50] E. Witten and D. I. Olive. Supersymmetry algebras that include topological charges. Phys. Lett., B78:97, 1978.

[51] F. A. Bais and W. Troost. Zero modes and bound states of the supersymmetric monopole. Nucl. Phys., B178:125, 1981.

[52] H. Osborn. Topological charges for $N=4$ supersymmetric gauge theories and monopoles of spin 1. Phys. Lett., B83:321, 1979.

[53] C. Montonen and D. I. Olive. Magnetic monopoles as gauge particles? Phys. Lett., B72:117, 1977.

[54] N. Seiberg and E. Witten. Electric - magnetic duality, monopole condensation, and confinement in $N=2$ supersymmetric Yang-Mills theory. Nucl. Phys., B426:19-52, 1994.

[55] N. Seiberg and E. Witten. Monopoles, duality and chiral symmetry breaking in $N=2$ supersymmetric QCD. Nucl. Phys., B431:484-550, 1994.

[56] F. Ferrari and A. Bilal. The strong-coupling spectrum of the Seiberg-Witten theory. Nucl. Phys., B469:387-402, 1996.

[57] A. Bilal and F. Ferrari. Curves of marginal stability and weak and strongcoupling BPS spectra in $N=2$ supersymmetric QCD. Nucl. Phys., B480:589-622, 1996.

[58] P. Forgacs, Z. Horvath, and L. Palla. Exact multi - monopole solutions in the Bogomolny-Prasad- Sommerfield limit. Phys. Lett., B99:232, 1981.

[59] Peter Forgacs, Zalan Horvath, and Laszlo Palla. Nonlinear superposition of monopoles. Nucl. Phys., B192:141, 1981.

[60] R. S. Ward. On selfdual gauge fields. Phys. Lett., A61:81-82, 1977.

[61] R. S. Ward. A Yang-Mills higgs monopole of charge 2. Commun. Math. Phys., 79:317-325, 1981.

[62] M. F. Atiyah and R. S. Ward. Instantons and algebraic geometry. Commun. Math. Phys., 55:117-124, 1977.

[63] M. F. Atiyah, N. J. Hitchin, V. G. Drinfeld, and Yu. I. Manin. Construction of instantons. Phys. Lett., A65:185-187, 1978. 
[64] F. A. Bais and H. A. Weldon. Exact monopole solutions in $S U(N)$ gauge theory. Phys. Rev. Lett., 41:601, 1978.

[65] D. Wilkinson and F. A. Bais. Exact $S U(N)$ monopole solutions with spherical symmetry. Phys. Rev., D19:2410, 1979.

[66] A. N. Leznov and M. V. Savelev. Exact monopole solutions in gauge theories for an arbitrary semisimple compact group. Lett. Math. Phys., 3:207$211,1979$.

[67] E. Witten. Some exact multipseudoparticle solutions of classical YangMills theory. Phys. Rev. Lett., 38:121, 1977.

[68] F. A. Bais, T. Tjin, and P. van Driel. Covariantly coupled chiral algebras. Nucl. Phys., B357:632-654, 1991.

[69] F. A. Bais and R. Sasaki. On the algebraic structure of selfdual gauge fields and sigma models. Nucl. Phys., B227:75, 1983.

[70] N. S. Manton. Instantons on a line. Phys. Lett., B76:111, 1978.

[71] W. Nahm. Multi - monopoles in the ADHM construction. Presented at 4th Int. Symp. on Particle Physics, Visegrad, Hungary, Sep 1-4, 1981.

[72] W. Nahm. All selfdual multi - monopoles for arbitrary gauge groups. Presented at Int. Summer Inst. on Theoretical Physics, Freiburg, West Germany, Aug 31 - Sep 11, 1981.

[73] M. C. Bowman, E. Corrigan, P. Goddard, A. Puaca, and A. Soper. The construction of spherically symmetric monopoles using the ADHMN formalism. Phys. Rev., D28:3100, 1983. 